\title{
Biomarkers indicative of blood-brain barrier disruption in multiple sclerosis
}

\author{
Emmanuelle Waubant* \\ UCSF MS Center, San Francisco, CA, USA
}

\begin{abstract}
Blood-brain barrier (BBB) disruption is one of the hallmarks of multiple sclerosis (MS). It is incompletely understood whether BBB disruption is the initial MS event leading to MS lesion formation or whether it is merely a consequence of cellular infiltration in the central nervous system (CNS). The presence of gadolinium enhancing $(\mathrm{Gd}+$ ) lesions on serial brain MRI scans is frequently used to evaluate BBB disruption. The presence of $\mathrm{Gd}$ enhancement has therefore been used as a reference for most works evaluating promising biomarkers of BBB disruption that are reviewed here. These promising biomarkers include cytokines and chemokines, and their receptors, cell surface markers, and matrix metalloproteinases and their natural inhibitors. At this time, none of these markers have been shown as sensitive as the presence of Gd enhancement to reflect BBB disruption. However, MRI scanning is not only unpractical and expensive; it may also under represent the overall extent of BBB disruption. Developing new MS biomarkers that are sensitive and specific for BBB disruption could 1) improve the monitoring of disease activity; 2) improve the monitoring of response to MS therapies which target BBB disruption; and 3) advance our understanding of dynamic MS processes participating in BBB disruption.
\end{abstract}

Keywords: Multiple sclerosis, blood-brain barrier, blood-spinal cord barrier, biomarker

\section{Introduction}

Blood-brain barrier (BBB) disruption is one of the hallmarks of multiple sclerosis (MS). It is incompletely understood whether BBB disruption is the initial MS event leading to MS lesion formation or whether it is merely a consequence of cellular infiltration in the central nervous system (CNS). In fact, disruption of vascular barriers in the CNS of patients with MS occurs not only at the level of the BBB, but also at the level of the blood-spinal cord barrier [1]. The presence of gadolinium enhancing $(\mathrm{Gd}+)$ lesions on serial brain and spinal cord MRI scans seems to be the most robust biomarker of BBB disruption at this time. Therefore, Gd enhancement has been used as a reference for most works evaluating promising biomarkers of BBB disruption. However, MRI scanning is not only unpracti-

${ }^{*}$ Corresponding author: UCSF Multiple Sclerosis Center, 350 Parnassus Avenue, suite 908, San Francisco, CA 94117, USA. Tel.: +1 4155142468 ; Fax: +1 4155142443 ; E-mail: waubant@itsa.ucsf. edu. cal and expensive; it may also under represent the overall extent of BBB disruption. Novel MS biomarkers which are sensitive and specific for CNS blood-barrier disruption and less expensive and easier to obtain than MRI scans with Gd injection, and which broadly reflect blood barrier disruption in the entire CNS could help 1) to better monitor disease activity; 2) to monitor response to MS therapies that target $\mathrm{BBB}$ disruption such as interferon- $\beta$ and natalizumab [2,3]; and 3 ) to advance our understanding of dynamic MS processes participating to BBB disruption.

\section{The blood-brain and blood-spinal cord barriers}

Because most of the work on CNS blood barriers pertains to the $\mathrm{BBB}$, this review will focus on biomarkers indicative of BBB disruption. The BBB is a complex structure constituted by the assembly of several cell types and matrix components $[4,5]$. The CNS microenvironment is important for neuronal function. The $\mathrm{BBB}$ is involved in its maintenance by protecting the 
brain parenchyma from abrupt metabolic changes and by maintaining apico-dorsal polarity. The selectively permeable $\mathrm{BBB}$ is rapidly responsive to physiological and pathological stimuli and plays a key role in maintaining the distinctive CNS metabolic and immunoregulatory homeostasis $[4,5]$. The barrier is present in a complex cellular system in which tight junctions between endothelial cells play a crucial role. Cells that compose the BBB in association with the basal laminae include endothelial cells, pericytes, perivascular microglia, and astrocyte processes. The unique properties of CNS endothelial cells compared with those present in other organs are induced by the neural environment during the development of the vascular system. Astrocytes that tightly appose end feet onto the abluminal side of brain capillaries seem to be important for the induction and maintenance of the endothelial barrier.

\subsection{The $B B B$ in $M S$}

The physiological low level of BBB exchange necessitates that cerebral endothelial cells (CEC) maintain multiple transporters for glucose and amino acids. CEC also express cholinesterases, monoamine oxidase, alkaline phosphatase and aromatic decarboxylases for catabolizing humoral transmitter substances [6]. $\gamma$ glutamyl transpeptidase appears to be another marker specific for CEC. In addition to these surface enzymes, CEC share common endothelial markers such as low density lipoprotein (LDL) and insulin receptors [6].

In the BBB, the tight and adherens junctions are the subcellular structures that maintain the restrictive properties of the BBB. BBB hyperpermeability has been linked to pathology in microvascular tight junctions (TJ). The evaluation of MS brains has shown that the TJ-associated protein zonula occludens-1 (ZO-1) has an abnormal pattern in oil-red O (ORO)-positive active plaques, affecting $42 \%$ of vessel segments, but this pattern is less frequent in ORO-negative inactive plaques $(23 \%)$, normal appearing white matter (NAWM) (13\%), and normal (3.7\%) and neurological controls (8\%) [6]. A similar pattern was found irrespective of the vessel size, supporting a causal role for diffusible inflammatory mediators.

\subsection{Causes of BBB disruption}

The BBB can be disrupted as a result of several processes. Massive migration of white blood cells (WBC) to the CNS or damage to blood vessel lining and surroundings (e.g., release of toxic factors in ischemia, or histamine in inflammation) can result in temporary $\mathrm{BBB}$ loss of integrity. On the contrary, neo vessels such as in cases of CNS tumor or vascular malformation may display an unusual fragility that can result in permanent increase of BBB permeability.

\subsection{Consequences of $B B B$ disruption}

A consistent MS feature is the transient or chronic loss of BBB impermeability. BBB disruption results in accumulation of serum proteins outside vessel walls [6$8]$ and may in turn facilitate cell migration to CNS. $\mathrm{BBB}$ disruption can be visualized in vivo by injection of gadolinium $(\mathrm{Gd})$, a contrast agent of small molecular weight that diffuses easily outside a BBB that has lost its integrity. Various degrees of disruption can be found from minimal and delayed Gd enhancement reflecting mild changes of permeability to massive and early Gd enhancement reflecting significant disruption $[8,9]$. The BBB breakdown may precede clinical signs, and constitute one of the first stages in the formation of most T2-bright areas, at least in relapsing forms of MS [9]. Gd enhancement in new or enlarging MS lesions lasts several weeks. Although the presence of Gd enhancement parallels a significant BBB leakage, it does not measure inflammation per se as once BBB permeability is restored, CNS inflammation may persist for a while.

Neurologists have initially attempted to identify biomarkers of MS activity by comparing various serum marker levels in patients identified as stable (no relapse) and those with recent relapses. Later, MRI scans demonstrated that macroscopical changes occurred significantly more often than clinical changes [9], thereby impairing the ability to identify biomarkers of disease activity when using purely clinical outcomes. In the past decade, the use of Gd enhancing MRI scans has enabled investigators to correlate more tightly several biomarkers to infraclinical MS activity measured by the presence of enhancement after Gd injection.

Several factors may limit our ability to use the presence of Gd enhancement as the ultimate marker of BBB disruption. The presence of Gd enhancement varies according to the dose of Gd that is administered, the time elapsed between Gd injection and image acquisition, and the severity of BBB disruption. This explains why mild chronic BBB leakage may not be detectable when MR images are obtained soon after the injection of Gd, as in progressive models of MS the presence of Gd enhancement may be delayed [10]. Further, it is unclear whether the best outcome measures of BBB disruption should be presence of Gd enhancement, number 
Table 1

Adhesion molecules and their ligands involved in diapedesis (modified from reference 15)

\begin{tabular}{|c|c|c|c|}
\hline Adhesion Molecules & Cellular Expression & Ligands & Main Function \\
\hline \multicolumn{4}{|c|}{ Immunoglobulin Superfamily } \\
\hline ICAM-1 & $\mathrm{E}, \mathrm{T}, \mathrm{B}, \mathrm{Ma}, \mathrm{Mi}, \mathrm{A}$ & LFA-1, MAC-1, CD43 & $\mathrm{T}$ cell migration \\
\hline VCAM-1 & $\mathrm{E}, \mathrm{Ma}, \mathrm{Pe}$ & VLA- $4, \alpha 4 \beta 7$ & $\mathrm{~T}$ cell and macrophage migration \\
\hline PECAM-1 & $\mathrm{E}, \mathrm{T}$ (naive), $\mathrm{Ma}, \mathrm{NK}$ & PECAM- $1, \alpha \mathrm{v} \beta 3$ & $\mathrm{~T}$ cell, natural killer and macrophage migration \\
\hline \multicolumn{4}{|r|}{ - } \\
\hline VLA-4 $(\alpha 4 \beta 1)$ & $\mathrm{T}, \mathrm{B}, \mathrm{Ma}$ & VCAM-1, FN, VLA-4, $\alpha 4 \beta 7$ & $\mathrm{~T}$ cell and macrophage migration \\
\hline LPAM-1 $(\alpha 4 \beta 7)$ & Memory T cells & VCAM-1, FN, MAsCAM-1 & Memory $\mathrm{T}$ cell migration \\
\hline LFA-1 $(\alpha \mathrm{L} \beta 2)$ & $\mathrm{Ma}, \mathrm{Mi}$ & ICAM-1, $-2,-3$ & $\mathrm{~T}$ cell and macrophage migration \\
\hline $\operatorname{Mac}-1(\alpha \mathrm{M} \beta 2)$ & $\mathrm{Ma}, \mathrm{Mi}, \mathrm{NK}$ & ICAM-1, FN, C3bi & Macrophage migration \\
\hline \multicolumn{4}{|l|}{ Selectins } \\
\hline E-selectin & $\mathrm{E}$ & sLx, ESL-1 & $\mathrm{T}$ cell rolling \\
\hline L-selectin & $\mathrm{T}, \mathrm{B}, \mathrm{Ma}$ & sLx, mucines & Rolling of T cell and macrophage \\
\hline P-selectin & $\mathrm{E}$ & sLx, PSGL-1 & Macrophage rolling \\
\hline \multicolumn{4}{|l|}{ Other } \\
\hline CD44 & $\mathrm{T}, \mathrm{B}, \mathrm{Ma}, \mathrm{A}, \mathrm{O}$ & MEC & $\mathrm{T}$ cell migration \\
\hline
\end{tabular}

Acronyms: C3bi: complement 3bi; ESL-1: L-selectin ligand; FN: fibronectine; ICAM-1: intercellular adhesion molecule-1; LFA-1: leukocyte function-associated molecule-1; LPAM-1: lymphocyte Peyer's patch adhesion molecule-1; Mac-1: macrophage glycoprotein associated with complement receptor function; MAdCAM-1: mucosal adressin cell-adhesion molecule-1; PECAM-1: platelet/endothelial cell-adhesion molecule-1; PSGL-1: P-selectin glycoprotein ligand-1; sLx: sialyl-Lewisx; VCAM-1: vascular-cell adhesion molecule-1; VLA-4: very late antigen-4.

A: astrocytes; B: B lymphocytes; E: endothelial cells; Ma: macrophages; Mi: microglia; NK: natural killer cells; O: oligodendrocytes; Pe: pericytes; T: T lymphocytes.

of enhancing lesions, volume of Gd enhancement or quantification of Gd enhancement signal over the entire brain. Second, most studies correlating biomarkers of interest to the presence of Gd enhancement rely purely on brain MRI scans. Therefore, the presence of spinal Gd enhancement in these instances is not part of the equation.

\section{Markers associated with BBB disruption}

Several markers have been studied for their ability to reflect $\mathrm{BBB}$ disruption in MS. Most of these are indirect markers of underlying biological processes participating in BBB disruption, e.g., markers of cell activation associated with BBB disruption except maybe for zonulin and endothelial microparticles $[11,12]$.

\subsection{Markers specific of BBB disruption}

An ideal marker of BBB disruption should be either specific for brain endothelium and therefore not found on other endothelial cell types, or be brain derived. Several markers may be specific for the BBB such as $\gamma$ glutamyl transpeptidase, cholinesterases, monoamine oxidase, alkaline phosphatase, aromatic decarboxylases, tight junction proteins (occludin, claudins, ZO-1 to -3 , cingulin, junctional adhesion molecules) and adherens junction proteins (VE-cadherin, catenins) [13].
There are no published reports on the levels of these markers in correlation with $\mathrm{BBB}$ disruption in MS. Regarding markers of brain damage and BBB disruption, only a few studies report increased levels of brain derived proteins such as $\mathrm{S} 100 \beta$ after brain trauma or stroke [reviewed in 14]. $\mathrm{S} 100 \beta$ is a protein primarily synthesized in the brain by astrocyte end feet processes and is quickly released from the brain into the blood when the BBB is disrupted [14]. While $\mathrm{S} 100 \beta$ appearance in plasma correlates well with an opening of the $\mathrm{BBB}$, it has also been shown to increase in plasma or $\mathrm{CSF}$ as a consequence of other disease processes not limited to the CNS. At this time, there is no evidence of such $\mathrm{S} 100 \beta$ increase related to BBB disruption in MS.

An ideal marker of BBB disruption should be measurable in serum or blood as opposed to CSF for ease of sampling and be virtually undetectable in healthy subjects. It should show distinct alterations in response to insults that are correlated with the severity of damage, represent disruption of $\mathrm{BBB}$ or blood spinal cord barrier, and be reproducible and inexpensive. Depending on the biomarker studied, a possible lag time between the increase of the biomarker level and the occurrence of BBB disruption must be considered [42]. In fact, some markers may relate to processes preceding $\mathrm{BBB}$ disruption while others may relate to processes occurring after BBB disruption has occurred [29]. 
Table 2

Adhesion molecules in MS and their relation to BBB disruption

\begin{tabular}{|c|c|c|c|c|}
\hline & Serum & $\mathrm{CSF}$ & Patients & References \\
\hline \multicolumn{5}{|l|}{ CD54 } \\
\hline \multirow{4}{*}{ sICAM-1 (E, T, B, Ma, Mi, A) } & $\uparrow$ & $\uparrow$ & Active disease (CS) & $16,17,18$ \\
\hline & & & Active clinical or MRI RRMS (LG) & \\
\hline & $\uparrow$ & & - levels in $\mathrm{RR}>\mathrm{SP}>\mathrm{PP}(\mathrm{LG})$ & 22 \\
\hline & & & $\begin{array}{l}\text { - high sICAM in patients with higher \# Gd+ } \\
\text { - Gd - patients had lower sICAM-1 }\end{array}$ & \\
\hline \multirow[t]{2}{*}{ sICAM-1 index } & $\uparrow$ & & - active RRMS (CS) & 21,23 \\
\hline & & & - index correlated with $\mathrm{Gd}+$ volume & \\
\hline \multicolumn{5}{|c|}{ 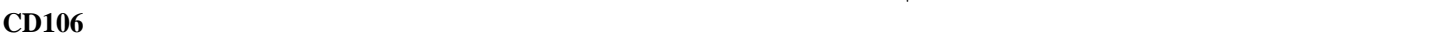 } \\
\hline \multirow[t]{2}{*}{ sVCAM-1 (E, Ma, Per) } & $\uparrow$ & & - levels in RR > SP MS (CS) & 19 \\
\hline & & & - levels higher in $\mathrm{Gd}+>\mathrm{Gd}-$ & \\
\hline sVCAM-1 index & $\uparrow$ & & Levels correlated with $\mathrm{Gd}+$ volume $(\mathrm{CS})$ & 23 \\
\hline \multicolumn{5}{|c|}{ 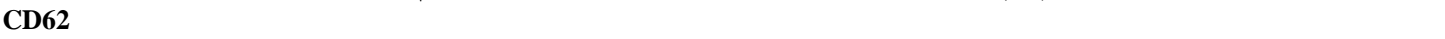 } \\
\hline \multirow[t]{3}{*}{ sL-selectin (T, B, Ma) } & $\uparrow$ & & - levels in RR > SP MS (CS) & 19 \\
\hline & & & - levels higher in $\mathrm{Gd}+>\mathrm{Gd}-$ & \\
\hline & $\uparrow$ & $\uparrow$ & $\begin{array}{l}\text { - levels higher in patients with } \mathrm{Gd}+ \\
\text { - levels correlated with size of } \mathrm{Gd}+\end{array}$ & 20 \\
\hline \multirow[t]{2}{*}{ sE-selectin (E) } & $\rightarrow$ & & - MS patients $(\mathrm{CS})$ & 18,19 \\
\hline & & & - Active clinical or MRI RR (LG) & \\
\hline \multicolumn{5}{|l|}{ Endothelial Microparticles } \\
\hline \multirow{2}{*}{$\begin{array}{l}\text { Insoluble PECAM-1 (CD31+) } \\
(\mathrm{P}, \mathrm{E}, \mathrm{Mo}, \mathrm{N})\end{array}$} & & $\uparrow$ & - higher in RRMS than controls (CS) & \\
\hline & & & $\begin{array}{l}\text { - higher in patients with exacerbation than remission } \\
\text { - higher in patients with } \mathrm{Gd}+\text { than } \mathrm{Gd}-\end{array}$ & 11,24 \\
\hline CD51 & & $\uparrow$ & - higher in RRMS than controls & \\
\hline (integrin $\alpha \mathrm{V} \beta 3$ ) & & & - same levels in patients with exacerbation and remission & \\
\hline$(\mathrm{E}, \mathrm{B}, \mathrm{Mo})$ & & & - same levels in patients with $\mathrm{Gd}+$ and $\mathrm{Gd}-$ & 11 \\
\hline
\end{tabular}

Abbreviations: LG: longitudinal, CS: cross-sectional, RR: relasping remitting, SP: secondary progressive. A: astrocytes; B: B lymphocytes; E: endothelial cells; Ma: macrophages; Mi: microglia; NK: natural killer cells; O: oligodendrocytes; Pe: pericytes; T: T lymphocytes.

\subsection{Non specific markers associated with $B B B$ disruption}

Multiple studies have evaluated the potential correlation of non specific markers involved in BBB disruption and are reviewed below. These include markers of cell adhesion, activation and attraction such as adhesion molecules, cytokines, chemokines and their receptors; and markers of diapedesis such as endothelial tight junctions, and matrix metalloproteases (MMPs) and their natural tissue inhibitors (TIMPs).

\subsubsection{Markers of cell adhesion, activation and attraction}

\subsubsection{Adhesion molecules as markers of $B B B$ disruption}

The activation of several cell types is a prerequisite for monocyte migration to the CNS. A tight regulation of various cell adhesion molecules (CAMs) is necessary for cell migration through tissues [see Table 1 adapted from 15]. Monocyte migration involves sequentially tethering, rolling via up-regulation of L-selectins (on leukocytes) and P-selectins (on endothelial cells), and monocyte arrest via up-regulation of E-selectins (on endothelial cells) and integrins (on leukocytes).

Several CAMs have been measured in the serum and CSF of patients with MS as potential markers of BBB disruption (Table 2) [11,16-24]. The main ones include soluble CD54 (ICAM-1), CD106 (VCAM-1), CD62 (L, $\mathrm{P}$ and $\mathrm{E}$ selectins), and endothelial microparticles (CD31 and CD51). Table 2 recapitulates published data for various markers in serum and CSF, the cells expressing or synthesizing these markers, and study design and patients characteristics.

Most CAMs are expressed by several cell types (see Tables 1 and 2), which decreases their potential specificity as markers of BBB disruption. The expression of CAMs at the cell surface is up-regulated in part by proinflammatory cytokines. CAMs are also shed in serum as soluble products. In vivo, soluble CAMs are detectable in serum and CSF and are believed to reflect cell surface expression levels. The factors underlying increased soluble CAM levels are unclear as increase in both production or membrane cleavage could result in these elevated CAM serum levels. The role of soluble CAMs remains unclear although it is suspected that they could inhibit cell adhesion. 
Table 3

Cytokines and their receptors as markers of BBB disruption

\begin{tabular}{llll}
\hline \multicolumn{2}{c}{ Blood } & Patients & References \\
\hline TNF and TNF receptor (TNF-R) & 26 \\
TNF $\alpha$ & $\rightarrow$ & RR/SP, no relation with MRI activity & 18 \\
TNF $\alpha$ mRNA & $\uparrow$ & Active clinical or MRI RR patients (LG) & \\
sTNF-Rp60 & $\uparrow$ & & 19 \\
sTNF-Rp60 & $\uparrow$ & Higher in patients with Gd+ than Gd- & \\
& & Higher in RR than chronic progressive (CS) & 27 \\
LN- $\alpha$ & $\rightarrow$ & No correlation with Gd+ & 27 \\
IL-2 and IFN- $\gamma$ & $\uparrow$ & - higher in RRMS, correlated with total \# Gd+ & 27 \\
IL-2 secreting cells & $\uparrow$ & - Trend only (LG) & \\
IFN- $\gamma$ secreting cells & $\uparrow$ & Unchanged with MRI activity & 28 \\
IFN- $\gamma$ mRNA & $\rightarrow$ & & \\
IL-12 & $\uparrow$ & Higher in RR/SP patients & \\
IL-12p40 mRNA & $\uparrow$ & Decreased when new MRI lesions appear \\
IL-12p35 mRNA & $\uparrow$ & Higher in RR when new lesions appear \\
IL-10 & $\uparrow$ & Higher when Gd+ resolves & \\
IL-10 mRNA & $\uparrow$ & 29 \\
IL-10 & &
\end{tabular}

LG: longitudinal, CS: cross-sectional, RR: relasping remitting, SP: secondary progressive.

$\mathrm{Gd}-$ : no gadolinium enhancing lesions.

$\mathrm{Gd}+$ : presence of gadolinium enhancing lesions.

Although several CAMs have been reported to be associated with BBB disruption [11,16-24], none are in fact specific or highly predictive of BBB disruption. While increased levels have been reported in patients with MS, there is a significant overlap with normal values or values in non-active MS patients. In fact, there is no data on sensitivity and specificity of these markers as they relate to BBB disruption. Interestingly, soluble E-selectin which is specific for endothelial cells is not elevated in patients with MS or increased during levels of disease activity $[18,19]$. Endothelial microparticles (EMPs) unlike other CAMs may reflect more directly the status of BBB endothelium in MS as cerebral endothelial cells activated by cytokines and chemokines shed EMPs into plasma [11]. These small membrane vesicles bear adhesion molecules from the activated parent endothelial cell. EMPs have been studied as markers of endothelial stress. Some of the markers carried by EMPs include PECAM-1 (CD31), CD51, endoglin (CD105), E-selectin, and VCAM-1. High plasma levels of EMP carrying CD31 are associated with the presence of contrast enhancing lesions on brain MRI scans in MS patients and may represent a new marker of disease activity [11]. Although the overlap between values of EMPs carrying CD31 in active MS patients and healthy individuals is much less than what is seen with other CAMs, the use of this marker is limited by the fact that EMPs can be increased in serum as the result of other inflammatory processes such as seen in patients with systemic lupus erythematosus.

\subsubsection{Cytokines and their receptors as markers of $B B B$ disruption}

Cytokines are small proteins that participate as extra cellular messengers in a wide range of host responses. MS has been associated with several immunoregulatory defects. The activity of Th2 cells which secrete the anti-inflammatory cytokines IL-4 and IL-10 appears reduced at the expense of an increased activity for Th1 cells which secrete increased amounts of the pro-inflammatory cytokines interferon- $\gamma$, TNF- $\alpha$ and IL-2 [for review see 25]. IL-12 appears to be another key pro-inflammatory cytokine in MS that is crucial for Th1 differentiation.

Similarly to CAMs, cytokines and their receptors are known to be involved in MS processes and have been evaluated as potential markers of BBB disruption (see Table 3) [18,19,26-29]. Several pro inflammatory cytokines appear to be correlated with BBB disruption.

As reflected in published data, cytokines and their receptors appear to be interesting markers for MS activity but none are highly specific or predictive of BBB disruption. Further, their levels fluctuate significantly within and between patients. Their levels in patients with MS also overlap with healthy controls as well as across disease types and courses. This is likely explained by the fact that changes in cytokine serum levels reflect a complex and intricate regulatory system for immune processes rather than mere BBB disruption alone. As a result, no specific cytokine or receptor level is used in clinical research or in clinical practice which would correlate with BBB disruption. 


\subsubsection{Chemokines and their receptors as markers of $B B B$ disruption}

Chemokines are released by several cell types throughout the body and define patterns of leukocyte migration to various organs guided by a restricted expression of their cognate receptors. For example, CCR5 participates in the directed migration of $\mathrm{T}$ cells into the CNS [30]. Chemokines have various effects beyond leukocyte recruitment such as the modulation of cytokine release and of nitric oxide production. The release of chemokines and the expression of their receptors are significantly modified by inflammatory conditions. Some chemokines contribute to inflammation such as monocyte chemoattractant protein 1 (MCP-1) whereas others such as fractalkine can limit inflammation.

Chemokines and their receptors are expressed differentially on various cell types and under various conditions. The regulation of a restricted number of chemokines and their receptors appears to regulate the traffic of inflammatory cells to the CNS and thereby could correlate with BBB disruption. Although increased numbers of CCR5-positive, IFN $\gamma$ - and TNF $\alpha$ secreting lymphocytes have been reported during acute MS exacerbations compared to controls, no MRI data is available for these patients to confirm a correlation with BBB disruption [31]. Recently, it was suggested that CXCR3 expression on CD8-positive T cells correlated with total T2 lesion volume, but no correlation was reported for Gd enhancing lesions with CXCR3, CCR2 and CCR5 expression on CD4- and CD8-positive T cells [32].

\subsubsection{Markers of diapedesis}

Markers of diapedesis include markers of BBB opening and of damaged cerebral endothelial cells, and markers that reflect the digestive ability of white blood cells to migrate to CNS tissues.

\subsubsection{Markers of BBB opening and damaged cerebral endothelial cells}

The main marker of an opening of endothelial tight junctions, e.g., the major keeper of vascular barrier permeability, is zonulin. Although not specific for the $\mathrm{BBB}$, zonulin is a protein modulating tight junctions, therefore playing a potentially crucial role in the modulation of BBB permeability in MS. One publication reports 1.5- to 3-times elevated serum levels in patients with relapsing remitting and secondary progressive MS compared to controls [12]. In this work, the highest levels of serum zonulin were reported in relapsing re- mitting patients who had Gd enhancing lesions. Further studies are needed to confirm the utility of this protein as a biomarker of BBB disruption in MS.

\subsubsection{Matrix metalloproteinases (MMPs) and their natural inhibitors (TIMPs)}

The balance between MMPs and their natural inhibitors (TIMPs) tightly regulates the digestion of the extracellular matrix and basement membranes, and thereby the migration of white blood cells to the CNS and other organs. MMPs are classified according to their substrates and structures and are reversibly inactivated when bound to a TIMP. MMPs and TIMPs participate in numerous physiological processes such as scaring, blastocyst implantation, and angiogenesis as well as pathological processes such as in rheumatoid arthritis, tumor infiltration, and metastatic dissemination [33].

MMP substrates include extracellular proteins such as collagen type I-VIII, gelatin, elastin, laminin but also myelin basic protein and several growth factors. MMP2 and -9 may be interesting markers of BBB disruption as they are the main MMPs for the degradation of collagen type IV and gelatin which are the main constituents of basal lamina. MMP-2 and -9 among others have been studied in several models for experimental allergic encephalomyelitis (EAE) [34-36]. Intravenous injection of MMP-9 was shown to open the BBB in animals [37] and MMP-9 levels are increased in EAE [34-36]. Human lymphocytes have been shown to produce several MMPs (MMP-2, -7, -9, -15, and -24 among others) and TIMPs [38-40] that are instrumental in $\mathrm{T}$ and $\mathrm{B}$ cell migration in vitro as they regulate digestion of the basal laminae $[37,38]$. These findings have prompted the study of MMP-9 and TIMP-1 in MS and their relation to BBB disruption as measured by the presence of Gd enhancing lesions on brain MRI scans [41-43]. Several remarkable findings are summarized below.

First, levels of MMP-9 were reported to be elevated in the serum of patients with relapsing remitting and secondary progressive MS compared to controls [4146]. Further, the main MMP-9 inhibitor, TIMP-1 does not seem to be increased in relation to MMP-9 suggesting an imbalance towards an increased digestive activity (Fig. 1) [41-43]. The use of the MMP-9/TIMP-1 ratio seems to provide a better measurement of proteolytic activity. However, as seen in Fig. 1, serum levels overlap significantly between MS patients and controls, even - although to a lesser extent - when using the ratio.

Second, MMP-9 serum levels were reported to be elevated in patients with $\mathrm{Gd}$ enhancing lesions as op- 


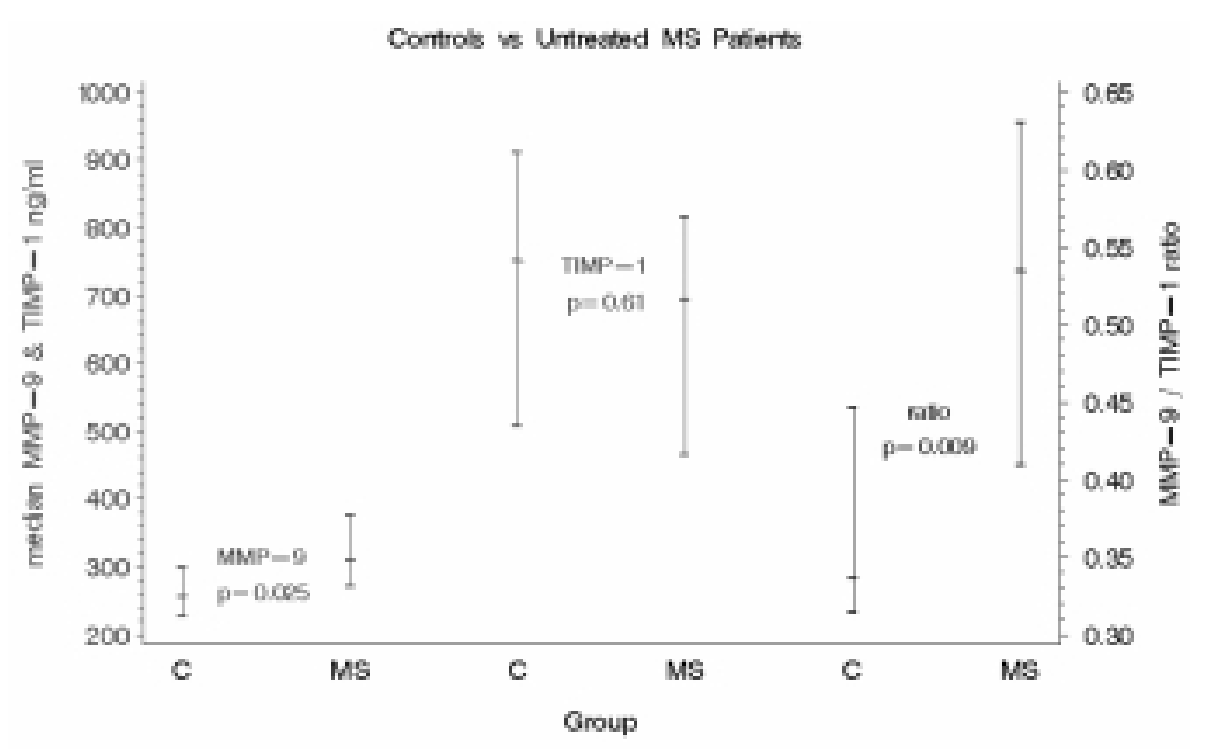

Fig. 1. Median serum levels for MMP-9 and TIMP-1, and MMP-9/TIMP-1 ratio in patients with relapsing remitting MS and controls [42].

Table 4

MMP-9/TIMP-1 predicts new gadolinium enhancement on subsequent brain MRI scan in RRMS [42]

\begin{tabular}{llc}
\hline & \multicolumn{1}{c}{$\mathrm{P}^{*}$} & Odds ratio * $(95 \% \mathrm{CI})$ \\
\hline High MMP-9 and TIMP-1 & 0.019 & $6.5(1.4,30.8)$ \\
Low MMP-9 and TIMP-1 & 0.043 & $4.7(1.0,21.3)$ \\
High MMP-9 and low TIMP-1 & 0.0006 & $21.5(3.8,121.4)$ \\
\hline
\end{tabular}

*versus reference category low MMP-9 and high TIMP-1.

Multivariate repeated measures logistic regression, levels dichotomized around median.

posed to those with no Gd lesions [43]. Further, higher levels of MMP-9 or lower levels of TIMP-1 seemed to predict the presence of Gd enhancing lesions the month of or the month after samples were obtained from patients (Fig. 2 and Table 4) [42,43]. This relation was found for MMP-9 and TIMP-1 as separate measures but appeared to be stronger when those measures were combined (Table 4) [42,43]. The predictive value of MMP-9 and TIMP-1 may be stronger in relapsing remitting versus secondary progressive MS $[42,43]$. No such relationship was reported for MMP-2 and TIMP2 [43].

Finally, interferon- $\beta$ therapy which reduces BBB disruption in MS appeared to result in lower levels of serum MMP-9, and even more significantly in higher levels of TIMP-1 in patients with relapsing remitting and secondary progressive MS, re-adjusting their MMP-9/TIMP-1 ratios towards normal values [43,47, 48].

While MMP-9 and TIMP-1 appear to be highly predictive of $\mathrm{BBB}$ disruption in relapsing remitting $\mathrm{MS}$,

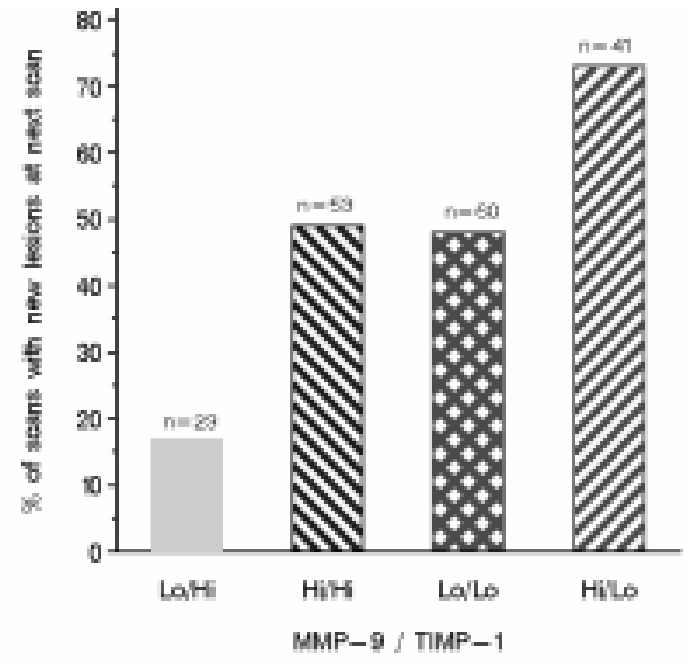

Fig. 2. Proportion of brain MRI scans with new gadolinium enhancement the month after MMP-9 and TIMP-1 measures [42]. Category groups include low MMP-9 and high TIMP-1 levels, high MMP-9 and high TIMP-1 levels, low MMP-9 and low TIMP-1 levels, and high MMP-9 and low TIMP-1 levels.

they do not seem very specific of BBB disruption per se as they may mostly reflect cell activation in general. The cells contributing to MMP-9 and TIMP-1 elevation include $\mathrm{T}$ and $\mathrm{B}$ cells, macrophages, and endothelial cells. Because MMP-9 and TIMP-1 levels fluctuate within and between patients, and are affected by infections, their use for a monitoring of the disease course in MS is difficult at best. It is interesting to note that the lack of a decrease of the MMP-9/TIMP-1 ratio dur- 
ing interferon- $\beta$ treatment may predict the occurrence of Gd enhancing lesions [43]. This suggests that the MMP-9/TIMP-1 ratio should be further evaluated as a surrogate of response to interferon- $\beta$.

\section{Conclusions}

To date, it has not been possible to identify biomarkers which would surpass Gd enhancement on brain MRI scans as measures of BBB disruption in vivo. Some would argue that alternative markers of BBB disruption might not be needed. Could such markers replace enhanced MRI scans or would they simply help us better understand disease processes? The need for an improvement of our ability to monitor the therapeutic response to agents that target BBB disruption appears to be the most critical.

Promising biomarkers of BBB disruption should be evaluated in conjunction with Gd activity on MRI scans obtained longitudinally as opposed to cross-sectional MRI studies or purely clinical outcomes, as this seems to be a more sensitive outcome. However, it remains unclear whether brain and spinal cord MRI scans should be combined to truly reflect BBB and blood-spinal cord barrier disruption.

It is unfortunate that published studies of MS biomarkers of BBB disruption have used various methodological approaches that have prevented a comparison of findings across studies. This argues for changing our practice. As new tools such as proteomics become available, we should evaluate promising markers of BBB disruption concomitantly in order to compare their sensitivity and specificity to predict BBB disruption. When appropriate, markers should be evaluated in parallel with their "inhibitor" or counterpart in order to assess a more global balance, such as IL10 and IL-12, or MMP-9 and TIMP-1. As no single marker appears to be as good as Gd enhancing MRI scans to monitor BBB disruption, we should likely focus on a combination of markers to improve sensitivity and specificity to detect BBB disruption. Improved understanding of BBB disruption is needed in MS. To advance our knowledge, measures of various biomarkers should be taken during clinical trials and natural history studies, or at least sample collection should be organized to allow for future such studies.

Finally, a specific effort should also be undertaken together with biostatisticians to advance the methodology of how best to evaluate promising biomarkers of BBB disruption. It appears that cross sectional studies of biomarkers of BBB disruption may be sub-optimal or even too crude in this day and age to take into account the complexity of MS processes, whereas longitudinal studies take into account fluctuations of several biological changes (e.g. serum and MRI) concomitantly $[28,29,42,43]$. This approach allows the use of biostatistical tools that also account for within-patient fluctuations $[29,42,43]$. The use of such models may permit concomitant analysis of several related or unrelated markers and may result in higher power to identify relevant markers of $\mathrm{BBB}$ disruption.

\section{References}

[1] P.M. Daniel, D.K. Lam and O.E. Pratt, Changes in the effectiveness of the blood-brain and blood-spinal cord barriers in experimental allergic encephalomyelitis, J Neurol Sci. 52 (1981), 211-219.

[2] IFNB Multiple Sclerosis Study Group. Interferon beta-1b is effective in relapsing-remitting multiple sclerosis: I. Clinical results of a multicenter, randomized, double-blind, placebocontrolled trial, Neurology 43 (1993), 655-661.

[3] D.H. Miller, O.A. Khan, W.A. Sheremata et al., A controlled trial of natalizumab for relapsing multiple sclerosis, $N$ Engl $J$ Med 348 (2003), 15-23.

[4] W. Risau and H. Wolburg, Development of the blood-brain barrier, Trends Neurosci 13 (1990), 174-178.

[5] W.A. Banks, Physiology and pathology of the blood-brain barrier: implications for microbial pathogenesis, drug delivery and neurodegenerative disorders, J Neurovirol 5 (1999), 538555.

[6] J. Kirk, J. Plumb, M. Mirakur and S. McQuaid, Tight junctional abnormality in multiple sclerosis white matter affects all calibers of vessel and is associated with blood-brain barrier leakage and active demyelination, J Pathol 201 (2003), 319-327.

[7] D. Gay and M. Esiri, Blood-brain barrier damage in acute multiple sclerosis plaques. An immunocytological study, Brain 114 (1991), 557-572.

[8] L. Claudio, C. Raine and C. Brosnan, Evidence of persistent blood-brain barrier abnormalities in chronic progressive multiple sclerosis, Acta Neuropathol 90 (1995), 228-238.

[9] A.G. Kermode, A.J. Thompson, P. Tofts et al., Breakdown of the blood-brain barrier precedes symptoms and other MRI signs of new lesions in multiple sclerosis, Brain 113 (1990), 1477-1489.

[10] C.P. Hawkins, F. Mackenzie, P. Tofts, E.P. du boulay and W.I. McDonald, Patterns of blood-brain barrier breakdown in inflammatory demyelination, Brain 114 (1991), 801-810.

[11] A. Minagar, W. Jy, J.J. Jimenez, W.A. Sheremata, L.M. Mauro, W.W. Mao, L.L. Horstman and Y.S. Ahn, Elevated plasma endothelial microparticles in multiple sclerosis, Neurology 56 (2001), 1319-1324.

[12] A. Minagar, M.G. Clemente, R.E. Kelley, J.S. Alexander and A. Fasano, Zonulin in multiple sclerosis: relation to subtypes of disease, Neurology 62(Suppl 5) (2004), A486.

[13] A. Minagar and S. Alexander, Blood-brain barrier disruption in multiple sclerosis, Multiple Sclerosis 9 (2003), 540-549. 
[14] N. Marchi, P. Rasmussen, M. Kapural, V. Fazio, K. Kight, M.R. Mayberg, A. Kanner, B. Ayumar, B. Albensi, M. Cavaglia and D. Janigro, Peripheral markers of brain damage and blood-brain barrier dysfunction, Restor Neurol Neurosci. 21 (2003), 109-121.

[15] J.J. Archelos and H.P. Hartung, The role of adhesion molecules in multiple sclerosis: biology, pathogenesis and therapeutic implications, Mol Med Today 3 (1997), 310-321.

[16] M.K. Sharief, M.A. Noori, M. Ciardi et al., Increased levels of circulating ICAM-1 in serum and cerebrospinal fluid of patients with active multiple sclerosis. Correlation with TNF $\alpha$ and blood-brain barrier damage, J Neuroimmunol 43 (1993), 15-22.

[17] H.P. Hartung, M. Michels, K. Reiner et al., Soluble ICAM1 serum levels in multiple sclerosis and viral encephalitis, Neurology 43 (1993), 2331-2335.

[18] P. Rieckmann, S. Martin, I. Weichselbraun, M. Albrecht, B. Kitze, T. Weber et al., Serial analysis of circulating adhesion molecules and TNF receptor in serum from patients with multiple sclerosis: cICAM-1 is an indicator for relapse, Neurology 44 (1994), 2367-2372.

[19] H.P. Hartung, K. Reiners, J. Archelos et al., Circulating adhesion molecules and tumor necrosis factor receptor in multiple sclerosis: correlation with magnetic resonance imaging, Ann Neurol 38 (1995), 186-193.

[20] R. Mossner, K. Fassbender, J. Kuhnen et al., Circulating Lselectin in multiple sclerosis patients with active, gadoliniumenhancing brain plaques, J Neuroimmunol 65 (1996), 61-65.

[21] M. Trojano, C. Avolio, I.L. Simone, G. Defazio, C. Manzari, F. De Robertis et al., Soluble intercellular adhesion molecule-1 in serum and cerebrospinal fluid of clinically active relapsingremitting multiple sclerosis: correlation with Gd-DTPA magnetic resonance imaging-enhancement and cerebrospinal fluid findings, Neurology 47 (1996), 1535-1541.

[22] G. Giovannoni, M. Lai, J. Thorpe et al., Longitudinal study of soluble adhesion molecules in multiple sclerosis: correlation with gadolinium enhanced magnetic resonance imaging, Neurology 48 (1997), 1557-1565.

[23] P. Rieckmann, B. Altenhofen, A. Riegel, J. Baudewig and K. Felgenhauer, Soluble adhesion molecules (sVCAM-1 and sICAM-1) in cerebrospinal fluid and serum correlate with MRI activity in multiple sclerosis, Neurology 41 (1997), 326-333.

[24] J. Losy, A. Niezgoda and M. Wender, Increased serum levels of soluble PECAM-1 in multiple sclerosis patients with brain gadolinium-enhancing lesions, J Neuroimmunol 99 (1999), 169-172.

[25] R. Hohlfeld, Biotechnical agents for the immunotherapy of MS: Principles, problems and perspectives, Brain 120 (1997), 865-916.

[26] B.W. van Oosten, F. Barkhof, P.E. Scholten, B.M. von Blomberg, H.J. Ader and C.H. Polman, Increased production of tumor necrosis factor alpha, and not of interferon gamma, preceding disease activity in patients with multiple sclerosis, Arch Neurol 55 (1998), 793-798.

[27] P.A. Calabresi, N.S. Fields, E.C. Farnon, J.A. Frank, C.N. Bash, T. Kawanashi, H. Maloni, S. Jacobson and H.F. McFarland, ELI-spot of Th-1 cytokine secreting PBMC's in multiple sclerosis: correlation with MRI lesions, J Neuroimmunol 85 (1998), 212-219.

[28] A.H. van Boxel-Dezaire, S.C. Hoff, B.W. van Oosten, C.L. Verweij, A.M. Drager, H.J. Ader, J.C. van Houwelingen, F. Barkhof, C.H. Polman and L. Nagelkerken, Decreased interleukin-10 and increased interleukin-12p40 mRNA are associated with disease activity and characterize different dis- ease stages in multiple sclerosis, Ann Neurol 45 (1999), 695703.

[29] E. Waubant, L. Gee, P. Bacchetti, R. Sloan, A. Cotleur, R. Rudick and D. Goodkin, Relationship between serum levels of IL-10, MRI activity and interferon beta-1a therapy in patients with relapsing remitting MS, J Neuroimmunol 112 (2001), 139-145.

[30] C. Trebst, T.L. Sorensen, Kivisakk et al., CCR1+/CCR5+ mononuclear phagocytes accumulate in the central nervous system of patients with multiple sclerosis, Am J Pathol 159 (2001), 1701-1710.

[31] T. Strunk, S. Bubel, B. Mascher, P. Schlenke, H. Kirchner and K.P. Wandinger, Increased numbers of CCR5+ interferon$\gamma$ - and tumor necrosis factor- $\alpha$-secreting T lymphocytes in multiple sclerosis patients, Ann Neurol 47 (2000), 269-273.

[32] R. Fox, P. Kivisakk, E. Fisher, B. Tucky, J.C. Lee, R. Rudick and R. Ransohoff, Biomarkers in MS: longitudinal analysis of chemokine receptor expression and MRi measures of injury implicates cytotoxic T-cell-mediated damage, Multiple Sclerosis 10 (Suppl 2) (2004), S108.

[33] H. Birkedal-Hansen, W.G.I. Moore, M.K. Bodden, L.J. Windsor, B. Birkedal-Hansen, A. DeCarlo and J.A. Engler, Metalloproteinases: a review, Crit Rev Oral Biol Med 4 (1993), 197-250.

[34] G.A. Rosenberg, M. Kornfeld, E. Estrada, R.O. Kelley, L.A. Liotta and W.G. Stetler-Stevenson, TIMP-2 reduces proteolytic opening of blood-brain barrier by type IV collagenase, Brain Res 576 (1992), 203-207.

[35] J.M. Clements, J.A. Cossins, G.M.A. Wells, D.J. Corkill, K. Helfrich, L.M. Wood, R. Pigott, G. Stabler, G.A. Ward, A.J.H. Gearing and K.M. Miller, Matrix metalloproteinase expression during experimental autoimmune encephalomyelitis and effect of a combined matrix metalloproteinase and tumor necrosis factor-alpha inhibitor, J Neuroimmunol 74 (1997), 85-94.

[36] B.C. Kieseier, R. Kiefer, J.M. Clements, K. Miller, G.M.A. Wells, T. Schweitzer, A.J.H. Gearing and H.-P. Hartung, Matrix metalloproteinase- 9 and -7 are regulated in experimental autoimmune encephalomyelitis, Brain 121 (1998), 159-166.

[37] S. Mun-Bryce and G.A. Rosenberg, Gelatinase B modulates selective opening of the blood-brain barrier during inflammation, Am J Physiol 274 (1998), 1203-1211.

[38] D. Leppert, E. Waubant, R. Galardy, N. Bunnett and S. Hauser, T cell gelatinases mediate basement membrane transmigration in vitro, J Immunol 154 (1995), 4379-4389.

[39] A. Alter, M. Duddy, S. Hebert, K. Biernacki, A. Prat, J.P. Antel, V.W. Yong, R.K. Nuttall, C.J. Pennington, D.R. Edwards and A. Bar-Or, Determinants of human B cell migration across brain endothelial cells, J Immunol 170 (2003), 4497-4505.

[40] A. Bar-Or, R.K. Nuttall, M. Duddy, A. Alter, H.J. Kim, I. Ifergan, C.J. Pennington, P. Bourgoin, D.R. Edwards and V.W. Yong, Analyses of all matrix metalloproteinase members in leukocytes emphasize monocytes as major inflammatory mediators in multiple sclerosis, Brain 126 (2003), 2738-2749.

[41] M.A. Lee, J. Palace, G. Stabler, J. Ford, A. Gearing and K. Miller, Serum gelatinase B, TIMP-1 and TIMP-2 levels in multiple sclerosis: a longitudinal clinical and MRI study, Brain 122 (1999), 191-197.

[42] E. Waubant, D. Goodkin, L. Gee, P. Bacchetti, R. Sloan, T. Stewart, P.-B. Andersson, G. Stabler and K. Miller, Serum levels of matrix metalloprotease-9 (MMP-9) and tissue inhibitor of MMP-type 1 (TIMP-1) predict MRI activity in relapsing multiple sclerosis, Neurology 53 (1999), 1397-1401.

[43] E. Waubant, D. Goodkin, A. Bostrom, P. Bacchetti, J. Hietpas, R. Lindberg and D. Leppert, IFN beta lowers MMP-9/TIMP-1 
ratio which predicts new enhancing lesions in patients with SPMS, Neurology 60 (2003), 52-57.

[44] R. Lichtinghagen, T. Seifert, A. Kracke, S. Marckmann, U. Wurster and F. Heidenreich, Expression of matrix metalloproteinase- 9 and its inhibitors in mononuclear blood cells of patients with multiple sclerosis, J Neuroimmunol 99 (1999), 19-26.

[45] Y. Galboiz, S. Shapiro, N. Lahat, H. Rawashdeh and A. Miller, Matrix metalloproteinases and their tissue inhibitors as markers of disease subtype and response to interferon-beta therapy in relapsing and secondary-progressive multiple sclerosis patients, Ann Neurol 50 (2001), 443-451.
[46] C. Avolio, M. Ruggieri, F. Giulani et al., Serum MMP-2 and MMP-9 are elevated in different multiple sclerosis subtypes, J Neuroimmunol 136 (2003), 46-53.

[47] M. Trojano, C. Avolio, G.M. Liuzzi, M. Ruggieri, G. Defazio, M. Liguori, M.P. Santacroce, D. Paolicelli, F. Giuliani, P. Riccio and P. Livrea, Changes of serum sICAM-1 and MMP-9 induced by rIFNbeta-1b treatment in relapsing-remitting MS, Neurology 53 (1999), 1402-1408.

[48] E. Waubant, L. Gee, K. Miller, G. Stabler and D. Goodkin, Interferon beta-1a may increase serum levels of TIMP-1 in patients with relapsing-remitting multiple sclerosis, J Interferon Cytokine Res 21 (2001), 181-185. 


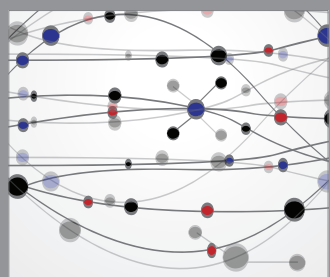

The Scientific World Journal
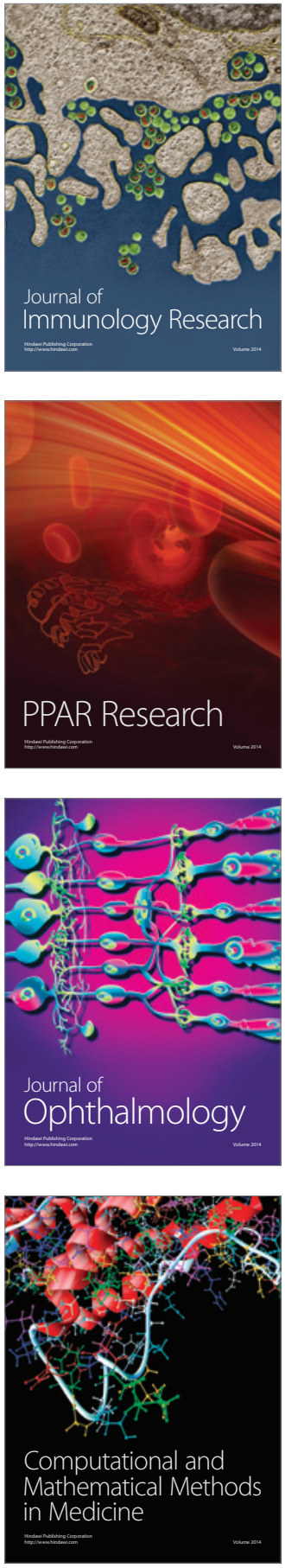

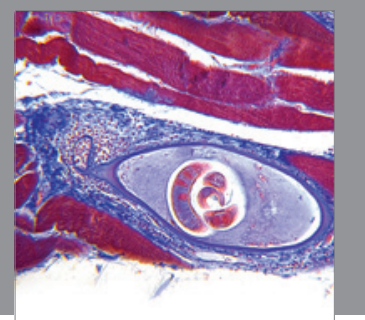

Gastroenterology

Research and Practice
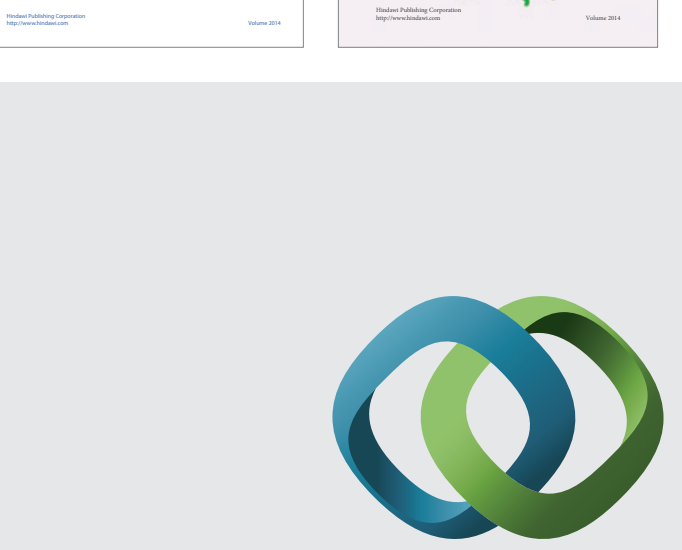

\section{Hindawi}

Submit your manuscripts at

http://www.hindawi.com
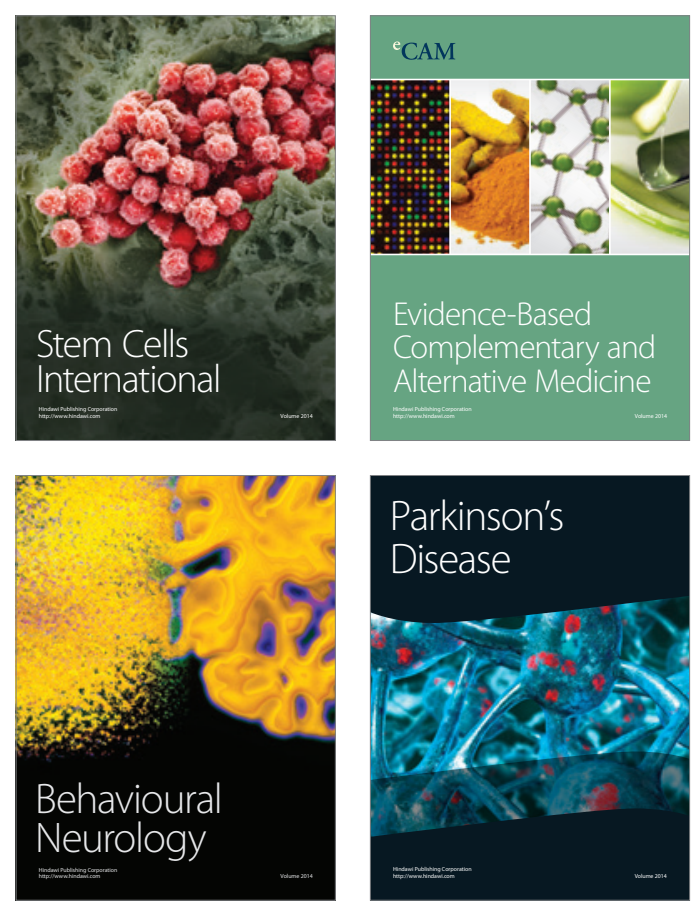

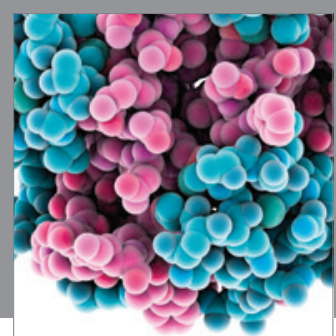

Journal of
Diabetes Research

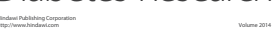

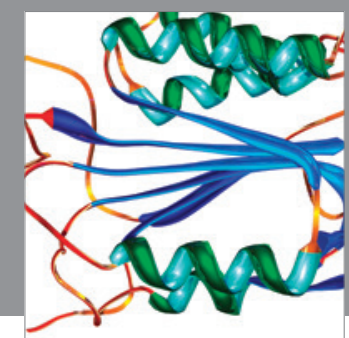

Disease Markers
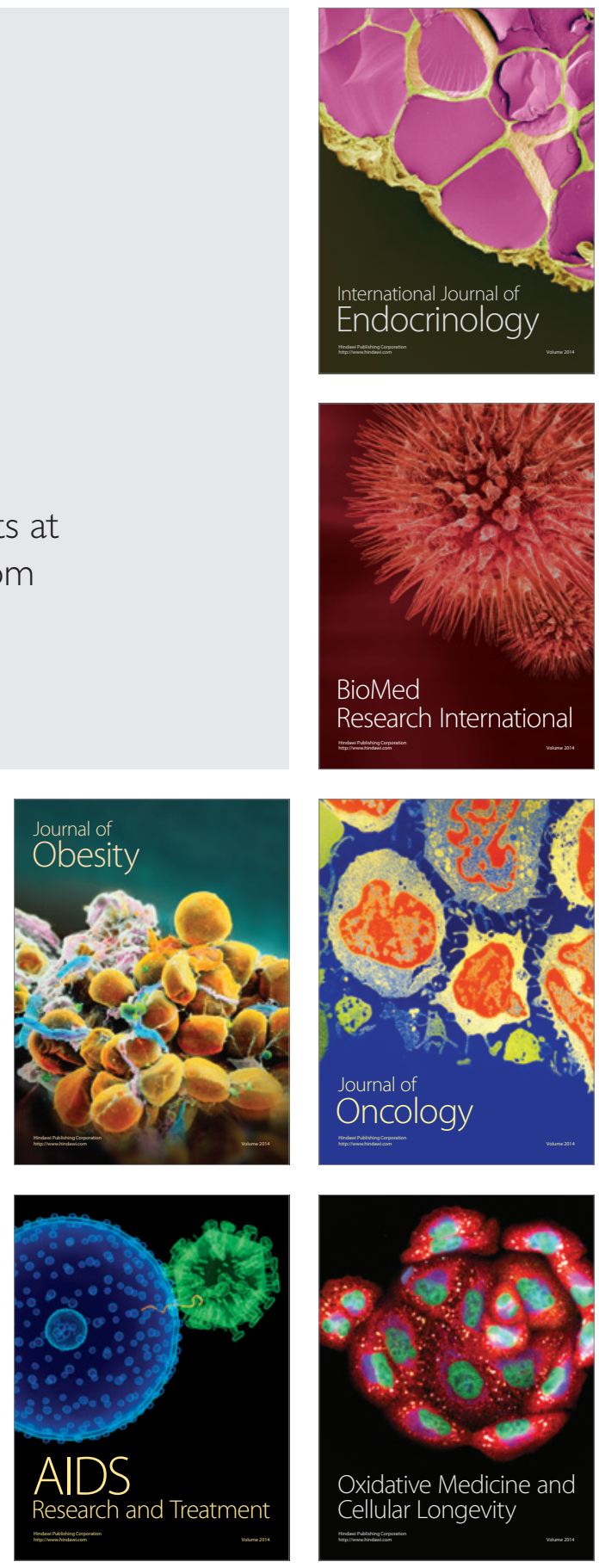